\title{
Sobre el parentesco histórico y estructural de las comparativas de igualdad y las consecutivas de intensidad
}

FERNANDO RIVERA CARDENAS

1. El análisis de las tradicionalmente llamadas subordinadas adverbiales impropias en español sigue planteando numerosos problemas. El primero, y básico, el de la caracterización de los diversos tipos oracionales que aquí se incluyen. Sin entrar en la discusión sobre si entre todos ellos existe una relación de interdependencia o interordinación como mantiene G. Rojo (1) -lo que, es cualquier caso, es difícilmente sostenible-, nos parece claro que tanto las comparativas como las consecutivas son, efecto, oraciones bipolares. La interdependencia entre los miembros de cada una de ellas viene señalada incluso desde el punto de vista constitucional por la presencia de las correlaciones tanto... como, tal...que, etc. Ninguno de los miembros puede aparecer solo sin que el resultado sea inaceptable, o sin que -n el supuesto de ser aceptable - haya transformado su naturaleza gramatical. Si en Estudia tanto como su hermano, conmutamos por cero el segundo miembro, obtendríamos $E$ studia tanto, que para ser aceptable precisaría un sintonema de anticadencia que la convertiría desde el punto de vista de la modalidad en una oración exclamativa ( $i E$ studia tanto!). En la estructura consecutiva Estudió tanto que se puso enfermo, efectuada la misma conmutación, el resultado se aproximaría más a la exclamativa, aun-

(1) Cfr, G. ROJO, Cláusulas y oraciones, Verba, anejo 14. Universidad de Santiago de Compostela, Vigo, 1978 y reseña de A. NARBONA en Studia Philologica Salmanticensia, n.o 3, 1979, 305-311. A. NARBONA, "Sobre las oraciones bipolares", Alfinge, 1, 1983, 121-139.

ALFINGE, 3 (1985), 115-124 
que sin llegar a ella. El tanto como término nuclear o tan como término adjunto en las consecutivas, aun siendo ascendente, no llega al que es peculiar de la exclamación. Por otra parte, la inaceptabilidad está fuera de duda si suprimimos el primer miembro en los dos casos: como su hermano, que se puso enfermo.

El hecho de ser bipolares ambos tipos y el de tener en común el primer término de la correlación - prescindamos de momento de lo suprasegmental - no puede hacernos perder de vista su distinta naturaleza sintáctica. El como de las comparativas introduce la comparación respecto a los sujetos (Estudia tanto como su hermano), verbos (EStudia tanto como se divierte), implementos (Bebe tanta cerveza como vino), aditamentos (Lo admiro tanto por su inteligencia como por su constancia en el estudio), etc. El que consecutivo, por el contrario, introduce una nueva predicación que es consecuencia o resultado de la intensidad o grado de cualidad del proceso que se enuncia en la llamada "oración principal".

2. Con independencia de tales valores plenamente diferenciados en sincronía -más adelante veremos casos en los que tal diferencia no es tan evidente-, la gramática generativo-transformacional ha intentado poner de manifiesto la relación entre los diversos tipos oracionales de carácter adverbial mediante complejos procesos de relativización. El caso que parece presentar menos dificultades teóricas es la historia derivacional de suboraciones adverbiales (2) introducidas por un adverbio relativo. En una oración como Yo trabajo tanto como él la estructura profunda sería (3):

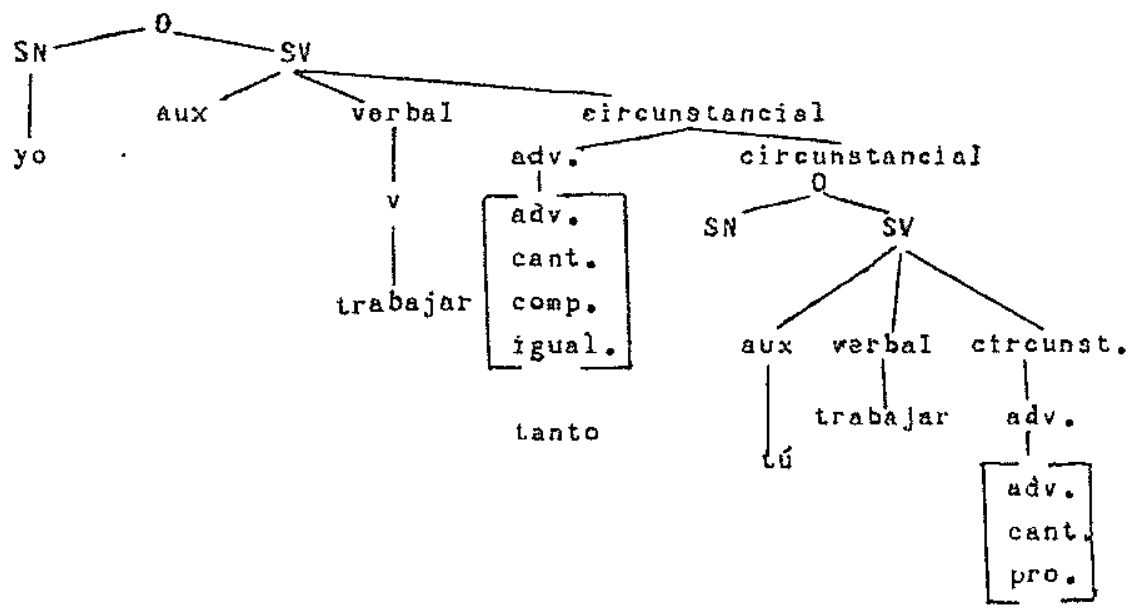

(2) Empleamos "suboración" para designar lo que muchos autores denominan 
El adverbio incrustado puede recibir el rasgo (trel.) en su matriz y trasladarse a la posición inicial. Como dice R. Hadlich (4), "el mismo tipo de la última regla que se requiere para especificar la forma superficial de los pronombres (que, quien, etc.), puede especificar fácilmente que como (llamado acertadamente adverbio relativo) es el verdadero relativo que hay que incrustar, cuando el antecedente es tanto". La deleción de la segunda aparición de trabajar sería una simpe transformación que eliminaría los elementos redundantes.

Dista de ser claro, sin embargo, que, como pretende R. Hadlich, una estructura profunda similar, pero cambiado el rasgo ( + igual.) por ( - igual.) dé como elemento de salida (out put) Yo trabajo más / menos que tú.

La principal objeción que puede presentarse es que en el caso de como puede admitirse con más facilidad el rasgo (t pro.) -aunque el hecho de no llevar antecedente, en el sentido convencional del término, sino el primer elemento de la correlación tanto, imponga ciertas reservas. Por el contrario, incluir tal rasgo en que comparativo supone atribuirle una naturaleza que choca al sentimiento del hablante-oyente.

El que de las comparativas, que según $\mathrm{E}$. Alarcos (5) es una conjunción cuya misión consiste en "unir segmentos equifuncionales de cuantificación diferentes" (/que/3), no puede ser en español actual un pronombre relativo, por más que se trate de un término que entre en correlación con más o menos del primer miembro. El hecho de que se

"proposición". Seguimos en ello a R. Lapesa: "Prefiero emplear el término suboración a usar proposición, porque las llamadas proposiciones, al carecer de autonomía dentro de una unidad supexior, no proponen nada" "Sobre dos tipos de subordinación causal", Estudios ofrecidos a E. Alarcos Llorach, III, Oviedo, 1978, p. 173).

(3) Cfr. Roger L. HADLICH, Gramática transformativa del español, Madrid, 1973, 284. En general, véase todo el capítulo XII, "La subordinación cir" cunstancial", 279-297, en el que esboza que no desarrolla con detenimiento- su hipótesis unificada para las adverbiales propias e impropias desde el punto de vista de la gramática generativo-transformacional.

(4) Ibid., 284-285.

(5) E. ALARCOS en "Español/que/", recogido en Estudios de gramática funcional del español, Gredos, Madrid, 2,a ed. aumentada, 197.8, 206, en nota a pie de página: "probablemente en las oraciones llamadas consecutivas se debe contar también con/que $/^{3}$ : tanto le insistieron que aceptó la oferta". C. Hernández Alonso cree que, en efecto, se trata de un /que/ ${ }^{3}$ ("El que español", $R F E$, L, 1967, 257-272), pero acaba llamándolo "conjunción re" lativa". Ese carácter relativo, con el mismo sentido que le da C. Hernández, lo encontramos en la Gramática Académica (Madrid, 1931): "se unen mediante el relativo neutro que, el cual se refiere a los antecedentes tanto, tan, tal, de modo, de manera, asi, expresos en la oración principal y callados rara vez" (párrafo 432). 
introduzca en la subcategoración de más los rasgos (+ cant.) y (-igual.) y en la de que (tcant.) y (+ pro.) no es, a nuestro juicio, razón suficiente para hablar de procesos de relativización. Según R. Hadlich (6), "la relativización tiene lugar, esencialmente, cuando se repite un antecedente de la oración matriz en la oración incrustada", pero no deja de ser relevante que los rasgos de subcategorización del antecedente que se repiten constituyen no una mayoría, sino una mínima parte de la totalidad de los rasgos que constituyen la matriz.

Menos claro todavía parece extender este principio al resto de las adverbiales, y sugerir la hipótesis de que las conjunciones circunstanciales, como él las llama, puedan generarse de manera similar, con la excepción de contados casos, que, además, "pueden analizarse, en su mayor parte como provistas de preposiciones, en vez de conjunciones, permitiendo clasificarlas entre los casos de subordinación nominal" (7); de esa forma, "todas las conjunciones adverbiales serían derivables de combinaciones de categorías simples. Todas las conjunciones adverbiales serían simples reflejos de ciertas condiciones de la estructura profunda" (8). Ello supone una generalización de escasa capacidad explicativa para la caracterización de cada tipo particular de subordinación adverbial. Serviría para las consecutivas de modo o manera (No me gustó la manera como lo hiciste) (9) y, con las dudas y reservas señaladas, para las comparativas de igualdad (Trabajo tanto como tú). Para los restantes casos esta hipótesis, que, esencialmente, hace de que una especie de traspositor universal, carece de la adecuada fundamentación teórica (10).

3. La hipótesis que comentamos quiere ser, a lo que parece, la explicitación desde los presupuestos teóricos de la gramática generativo-transformacional de un antiguo planteamiento sugerido por la lingüística histórica para la época del nacimiento de las lenguas romances. Nos referimos a QUOD como partícula universal de subordinación. Hagamos unas mínimas considexaciones históricas, que pueden servir para arrojar alguna luz sobre la subordinación en general $\mathrm{y}$, en particular, sobre las comparativas de igualdad y las consecutivas de intensidad.

A finales del Imperio, al producirse la ruina de la declinación,

(6) Cfr. Roger L. HADLICH, op. cit., 283.

(7) Ibid., nota 107.

(8) Ibid., 288.

(9) Cir. A. NARBONA JIMENEZ, Las proposiciones consecutivas en español medieval, Universidad de Granada, 1978,172 y 215 y ss.

(10) Cfr. C. HERNANDEZ ALONSO, Gramática funcional del español, Gredos, Madrid, 1984. Particularmente, los transpositores del llamado grupo II, 239-244. 
la forma QUOD es la única forma que sobrevive del relativo, desempeñando sus funciones propias y las que antes estaban asignadas a las otras formas. Se convierte, pues, en un relativo con valor universal, con antecedente en cualquier género o número: Form. And. $193 \mathrm{rem}$ superius nominata, quod a uobis delegabit, ibid. 818 diuina omnia, quod hic aguntur, ibíd. 1974 illud imprimitas, quod plurima sunt et nobis oporiunum salutem in Domino optamus uobis, Mulomed. 392 (11926) ilia ubi cinguntur intestina, quod quidam sapientiam uocant, Acta Andreae 575 Fecitque ibi magna mirabilis quod nullus ualuit facere nisi solus deus, etc. Por otra parte, la conjunción QUOD fue adquiriendo los más variados sentidos: temporal, consecutivo, completivo, causal, etc. Las interferencias entre el "relativo indeclinable", que podía llevar cualquier antecedente, y el QUOD conjunción tuvieron que ser muy fuertes en la lengua latina tardía. En los siguientes ejemplos -por citar algunos- se observa cómo el sentido relativo y el sentido causal pueden considerarse posibles: Form. Senon. Cartae, Appendix 1 d (20931): haec cartola, quem infantes iuos, quod naturales sunt, in legitima hereditate secundem lege instituisse y Lex Cur. IV, 13 (34720): in causas inter priuatos homines, quod publice actiones non sunt, nemini licet ad extraneum iudicem ipsa causa in iudicio mittere (11). Y así es razonable suponer que la comunidad de hablantes, poco dada a distinciones y precisiones, no expresó de forma diferenciada la subordinación causal y la relativa - por seguir con el ejemplo aducido. Tal confusión, desde luego, no sería privativa de estas subordinaciones, sino que se extendería a finales, temporales, etc., etc. Como señala J. Herman, "quod en lui-même n'est plus que le signe abstrait d un rapport de subordination et dont la valeur précise dépend entièrement du contexte dans lequel il apparait" (12). Concretamente, en el caso del desplazamiento de $u t$ consecutivo (talisque illum ultio consequator diuina que omnes uidentes terreant et audientes contremescant $)(13)$, pudiera formularse la hipótesis de que quod o que ofrecían un sentido más préciso, menos sujeto a complejos condicionamientos sintácticos. Se trata, en definitiva, de un proceso de simplificación entre los diversos tipos oracionales subordinados, aunque, por otra parte, se procede a un reagrupamiento de nuevos enlaces que, en un largo proceso evolutivo, culminará en un mayor enriquecimiento de las posibilidades expresivas.

(11) Cfr. J. HERMAN, la formation du système roman des conjoncions de subordination, Akademie-Verlag, Berlín, 1963, 67.

(12) Ibid., 70 .

(13) Citado por J. BASTARDAS PARERA, Particularidades sintácticas del latin medieval (Cartularios españoles de los siglos VIII al XI), CSIC, Barcelona, $1953,192$. 
Cabría plantearse la naturaleza del proceso: la forma $Q U O D$, sentida como conjunción, desplaza a la conjunción UT, o bien, sentida confusamente como relativo, realiza tal desplazamiento. Parece probable que en principio existiera la dualidad de sentido lingüístico, pero que, conforme iba avanzando la ruina del sistema latino, el hablante llegara, en un momento dado, a una confusión total. QUOD que se habría convertido en un elemento "polifuncional", en un elemento sobrecargado de funciones de difícil delimitación con fecuencia.

Es posible que, así las cosas, predominara "el sentido del relativo", aun cuando fuera UT el elemento desplazado. En tal caso parece lógico que, por así decir, el hablante buscara un antecedente para él. A. Narbona ha estudiado esta posibilidad. Apoyándose fundamentalmente en la confusa situación de la forma $Q U O D$ y en la igualdad.de los primeros términos de las correlaciones de las comparativas de igualdad y las consecutivas de intensidad (tan...que, tanto...que, tal...que, tan...como, tanto...como) llega a la hipótesis de que en toda consecutiva de intensidad subyace, por así decir, una comparativa de igualdad:

Corrió tanto como $X$. $X$ perdió el aliento.

Corrió tanto que (el cual) perdió el aliento.

donde $\mathrm{X}$ tendría como rasgos subcategorizadores, entre otros, ( - def., + univ.). La comparación, que no es explícita, no se da en el nivel de superficie. Aquí sólo aparece tan o tanto con tonema ascendente y que consecutivo. Como se observará, en el proceso se ha producido un cambio de sujeto: en la comparación no explícita $\mathrm{X}$ es el sujeto de perdió en la oración $X$ perdió el aliento; después, tanto perdió como corrió llevarán el mismo sujeto. Como es claro, el planteamiento habría que modificarlo si se tratara del caso siguiente:

Ella corrió tanto co mo $X . Z$ se quedó detrás.

Ella corrió tanto que él se quedó detrás,

que presentaría una historia derivacional más compleja, puesto que él sólo podría insertarse después de que que hubiera transformado su naturaleza, pasando a conjunción consecutiva, $\mathrm{y}$, uniendo, como señala E. Alarcos, elementos equifuncionales de cuantificación diferente. El encarecimiento propio de las consecutivas, del que habla A. Bello (14) se produciría precisamente cuando que se siente ya como conjunción.

Propiamente, A. Narbona no supone una estructura profunda en

(14) A. BELLO, Gramática de la lengua castellana, editada con las notas de R.J. CUERVO, Sopena-Argentina, Buenos Aires, 8. a ed., párrafo 1062: "tal que determina la cualidad encareciéndola; y lo hace por medio de una circunstancia que no tiene semejanza con ella". 
términos chomskyanos. Se refiere, únicamente a •una construcción "que subyace", por así decir, y que de modo confuso tuvo que sentir la comunidad de hablantes, en un proceso innovador colectivo, al emplear una forma que originaria y fundamentalmente era un relativo.

Son muy distantes las actitudes teóricas de A. Narbona y R. Hadlich. Es cierto que Narbona cita a Hadlich a propósito de las consecutivas de manera (15), pero no lo es que su hipótesis sobre la comparación subyacente a las consecutivas de intensidad se relacione directamente con las propuestas del autor norteamericano. Narbona mantiene que QUOD que tuvo en principio valor de relativo -n lo que, por diversos caminos, podría estar de acuerdo con Hadlich-, pero en seguida señala el cambio de naturaleza a conjunción. Su hipótesis arroja luz sobre la diacronía, pero inmediatamente conecta el pasado con el presente sincrónico. Diacronía y sincronía no son, en efecto, polos irreconciliables, y la investigación histórica puede contribuir a explicar determinados fenómenos de caracter sincrónico.

4. Nos resulta difícil -tal vez hasta no sea posible- verificar que el paso propuesto por Narbona se hubiera producido en un momento dado. Parece, con todo, lo más razonable. Pero, en cualquier caso, no deja de ser altamente significativo a favor de su hipótesis la confluencia tanto diacrónica como sincrónica que se produce entre ambos tipos oracionales, las comparativas de igualdad y las consecutivas de intensidad.

Señala F. Krüger que la exclamativa iEs de lindo! - e igual podríamos decir de ${ }_{j} E$ s tan lido! - encuentra a veces una especie de desarrollo "de la tensión emotiva propia del tal giro elíptico". Ese desarrollo aparece "en dos formas distintas, pero -debido a su objeto común- psíquicamente afines: la comparación o la subordinada consecutiva, la cual, además, puede contener una comparación. (...) Tanto la una como la otra contribuyen a agregar al adjetivo (...) una nota concreta, a veces hasta cierta plasticidad o un nuevo elemento reforzativo" (16) Así sucedería en

(1) Es tan hermosa como un sol.

(2) Es tan hermosa que es un sol.

(3) Es tan listo como un lince.

(4) Es tan listo que es un lince.

(15) A. NARBONA, op. cit., 172. Vid. nota 8.

(16) F. KRƯGGE, El argentinismo iEs de lindo...!, CSIC, Madrid, 1960, 131. Vid. en general, los párrafos 75 y 76 . Desde el punto de vista expresivo, las aportaciones de Krüger son fundamentales para nuestra hipótesis. Aquí se señalan por primera vez un cierto tipo de conexión -si bien solamente expresivo-entre las construcciones que nos ocupan. 
La unión "psíquica" de la que habla F. Krüger no impide, desde luego, tener presente que las estructuras están perfectamente diferenciadas: unas son comparativas ( 1 y 3 ) y otras consecutivas (2 y 4). Sería inaceptable decir Es tan listo que un lince, pues faltaría precisamente la predicación que existe en cualquier consecutiva como resultado de la intensidad o el grado de cualidad de un proceso. Con todo, esa unión "psíquica" señala un primer grado de acercamiento a la verificación de las conexiones que en el plano expresivo pueden mostrar estos tipos oracionales.

4. 2. Desde el punto de vista histórico puede observarse cómo el segundo término de la correlación ofrece un carácter vacilante en algunas ocasiones. Así, en

$E$ dizen que traya en esta galea tantas joyas e tan preciadas las quales fasta en aquel tiempo nunca fueron vistas (Leóm. CXCV-296).

puede entenderse que no se cierra la correlación, o bien que las cuales es un relativo con sentido consecutivo equivalente a que (tan preciadas...que), lo que vendría a confirmar la primigenia naturaleza relativa del elemento que encabeza el segundo miembro de la correlación, en la línea apuntada por A. Narbona. Este relativo puede ir, asimismo, precedido de preposición:

Si fuesen los bues o las bestias tales con ke pueda omne labrar (Crestomatia, p. 114, doc. III); Vidi y tales cosas por qui so muy pagada (Sta. Oria 154d); Afe yo trametré viento e oyra tal palabra por que tornará a su tierra e yl fara morir a espada (Faz. 155).

Otras veces, también en la lengua medieval, en la que pugnan por consolidarse estos procedimientos expresivos, encontramos estructuras en las que aparecen seguidos como y que con sentido comparativo-consecutivo:

E otra tentaçion, floxa en paresçer, / Demuestra se tan flaca como que non ha poder / para donar (Rim. Pal. E 1655).

Igualmente reveladoras son aquellas estructuras en las que aparece como introduciendo una suboración con sentido híbrido, entre comparativo-modal, con su antecedente en manera, y consecutivo, por llevar el primer término de la correlación tal. La lengua medieval "nada sujeta a criterios de correción normativa (...) presenta constantemente soluciones que rompen los modelos sintácticos 'normales" "(17) Unas veces por falta de habilidad, otras porque el idioma -todavía con poca andadura- no ofrece soluciones consolidadas y otras muchas porque la sintaxis se doblega a la intención expresiva del hablante. $\mathrm{Y}$ así leemos en los Documentos lingüisticos de R. Menéndez Pidal: 
De tal manera aredraremos como vos don Adam $₹$ uestra mugier tenientes seades toda via atodas maneras (p. 452, 23-25, doc. 336); De tal manera riedre zuos la faga sana, yo o qui lo mio eredare, como uos los compradores connombrados, o qui lo uestro heredare, finquedes por siempre con uestra compra sobredicha en todas manera (p. 455, 22-25, doc. 339); De tal manera riedre $\tau_{\text {uolla faga }}$ sana como uos el comprador $\tau$ quien lo uestro heredare finquedes por siempre con uestra compra sobredicha entodas maneras, sin contralia nenguna (p. $456,20-22$, doc. 340 ); De tal manera redremos como uos los compradores $\mathbf{t}$ quien lo uestro heredare ffinquedes por siempre con uestra compra sin contralla (p. 462, 20-22,

$Y$ otro caso en el que falta $t a l$, pero que puede clasificarse con los anteriores:

De manera redremos zuos le fagamos sano commo uos los compradores quien lo uestro heredare finquedes por siempre con esta compra sin contralla (p. 463, 19-21, doc. 348).

Gjemplos similares se encuentran en época más tardía. Así en la Crónica del Rey D. Pedro:

(mandó que) ... pusiese tal recabdo como ella non pudiese por ninguna manera partir de alli del Alcazar fasta que el Rey ordenase do lo auia de tener presa (IX, 464a); $E$ si algunas otras cosas oviere de librar entre el e vos, nos con la merced de Dios entendemos ponerlas en tal estado como vos seades contento (X, 555b).

Por otra parte, como señalaba certeramente A. Narbona, "muchas veces una estructura formalmente consecutiva no es más que una variante más expresiva de la comparación" (18):

Aqui veo atal cosa que nunca vi tan grande (Roncesv. 35); $E$ mando fer Pascua a fijos de Israel, e fezieronla tan rica mientre que non fue tal alta mientre en tierra de Jherusalen (Faz. 159).

Particularmente reveladoras son las estructuras en las que como y que resultan intercambiables:

Non ha dulçe cosa, como la segurança, ni miel atán sabrosa que paz e amistança.

(Proverbios de Sem Tob, 1039-1042).

Ejemplos de este tipo siguen apareciendo todavía en el español clásico. Así, en el Guzmán de Alfarache:

Tanto me daba trabajar en esto que en esotro. (II, 71, 6).

En español actual permanecen, si bien en escasa proporción, ca- 
sos similares al del Guzmán:

Tanto me da ocho que ochenta.

Tanto me da escribìr que no escribir.

Tanto me importa ir al cine que quedarme en casa.

Con todo, los ejemplos podrían multiplicarse. La sustitución de que por como no altera el significado, lo que hace que las suboraciones introducidas por que o como puedan considerarse equivalentes en tales casos.

El parestesco histórico entre ambas estructuras quedaría de manifiesto, a nuestro modo de ver, en estas oraciones en las que pueden emplearse uno u otro nexo sin que se altere el significado. Constituirían una especie de reliquia -en el sentido etimológico de la palabra-, una tertia via, solución híbrida e imprecisa entre dos procedimientos expresivos que acabaron consolidándose por caminos diferentes. 